\title{
Avaliação da Percepçáo Corporal em Pacientes Pós-Acidente Vascular Cerebral (AVC)
}

\author{
Evaluation of Corporal Perception In Post Stroke Patients \\ Ana Paula Chaves Dalpian', Magali Teresinha Quevedo Grave2, \\ Eduardo Périco ${ }^{3}$
}

\section{RESUMO}

O Acidente Vascular Cerebral (AVC) é uma condição patológica que tem como principal consequência a hemiplegia, anormalidade caracterizada pela paralisia e/ou paresia do lado contralateral à lesão encefálica. A hemiplegia afeta a percepção corporal e o alinhamento, alterando toda a cadeia muscular e postura corporal contra a gravidade. Na síndrome de Pusher (SP), um conjunto de características leva o paciente a realizar uma forte inclinação do corpo em direçâo ao lado plégico. O objetivo da pesquisa, caracterizada como exploratória, descritiva, de caráter quantitativo, foi avaliar o grau de comprometimento da percepção corporal em pacientes pós-AVC, atendidos na Clínica Escola de Fisioterapia da Univates/Lajeado/RS, durante o semestre B de 2012, cujo instrumento de coleta de dados foi a Clinical Scale for Contraversive Pushing (CSCP), protocolo disposto de três etapas: em sedestação, ortostase e durante movimento ativo do membro superior (MS) não afetado. Dos treze pacientes avaliados (8 homens e 5 mulheres), os resultados apontam que cinco apresentam alteração da percepção corporal. Sete tem a metade corporal esquerda comprometida e em oito o AVC foi isquêmico. Conclusáo. a SP é um distúrbio presente nestes sujeitos, a CSPS é um protocolo de fácil aplicação, que favorece a identificação e gravidade desta alteração.

Unitermos. Acidente Vascular Cerebral, Hemiplegia, Postura.

Citaçáo. Dalpian APC, Grave MTQ, Périco E. Avaliação da Percepção Corporal em Pacientes Pós-Acidente Vascular Cerebral (AVC).

\begin{abstract}
The Cerebral Vascular Accident (CVA) is a pathological condition whose main consequence hemiplegia abnormality characterized by paralysis and/or paresis of the contralateral brain injury. Hemiplegia affects body awareness and alignment, changing the whole chain muscle and body posture against gravity. In Pusher syndrome (SP), a set of characteristics leads patients to make a strong body tilt toward the paralyzed side. The purpose of the research, characterized as exploratory, descriptive, quantitative character, was to assess the degree of impairment of body awareness in post-stroke patients, treated at the Clinical School of Physiotherapy Univates / Paved / RS during the semester B 2012 whose instrument of data collection was the Clinical Scale for Contraversive Pushing (CSCP), the protocol provided three stages: sitting position, standing position and during active movement of the upper limb (UL) unaffected. Of the thirteen patients evaluated ( 8 men and 5 women), the results show that five have changes of body awareness. Seven have impaired left body half and eight strokes were ischemic. Conclusion. SP is a disorder present in these subjects, the CSPS is a protocol for easy application, which favors the identification and severity of this change.
\end{abstract}

Keywords. Stroke, Hemiplegia, Posture.

Citation. Dalpian APC, Grave MTQ, Périco E. Evaluation of Corporal Perception In Post Stroke Patients.
Pesquisa realizada na Clínica-Escola do Centro Universitário Univates, Lajeado-RS, Brasil.

1.Fisioterapeuta, Centro Universitário Univates, Lajeado-RS, Brasil.

2.Fisioterapeuta, Doutora em Ciências da Saúde PUCRS. Professora dos Cursos de Fisioterapia e Educação Física do Centro Universitário Univates, Lajeado-RS, Brasil.

3.Biólogo, Doutor em Ecologia pela Universidade de São Paulo (USP), Programa de pós-graduaçáo em Ambiente e Desenvolvimento, Centro Universitário Univates, Lajeado-RS, Brasil.
Endereço para correspondência: Magali T. Q. Grave Rua 13 de Maio 230/301 CEP 95880000, Estrela-RS, Brasil. E-mail: mgrave@univates.br 


\section{INTRODUÇÃO}

O Acidente Vascular Cerebral (AVC) é a principal causa de mortes por doenças cerebrovasculares no Brasil, o que torna cada vez maior a incidência deste tipo de pacientes em clínicas de fisioterapia ${ }^{1}$.

Popularmente conhecido como derrame, o AVC, que acompanha lesóes hemorrágicas ou isquêmicas, tem como causas a aterosclerose, trombose cerebral, êmbolos cerebrais, aneurisma etc. Como fatores de risco, pode-se citar hipertensão arterial, diabetes e idade, com uma ampla lista de consequências, que vão desde dor, déficits visuais, déficits somatossensitivos, distúrbios de fala e linguagem, disfagia, alteração da cognição e déficits motores, dentre os quais se destaca a hemiplegia, anormalidade que se caracteriza pela paralisia e/ou paresia do lado contralateral à lesão encefálica ${ }^{2-4}$.

A hemiplegia constitui-se basicamente na perda da motilidade voluntária dos segmentos corporais da metade do corpo comprometidos pela lesão. É uma situação grave e incapacitante, tornando-se um desafio à reabilitação. No caso do AVC, a forma mais comum é a hemiplegia espástica, gerando aumento do tônus de grupos musculares, levando a deformidades e contraturas, quando não tratada $^{5,6}$.

A prevenção se embasa na modificação dos fatores de risco, como regulagem da pressão arterial sistêmica, dieta, exercícios físicos etc ${ }^{2}$. O sistema nervoso central é constantemente alimentado por informações (necessárias para ajustes do funcionamento do organismo) e de atividade dos sistemas sensoriais, tais como a percepção. A somestasia (sistema somestésico) é um sentido que contrasta com visão, olfato, gustação, audição e equilíbrio. É uma sensibilidade geral que, subdividida, tem duas facetas, sendo que uma delas, a Somestasia Proprioceptiva, é voltada para a parte da sensibilidade externa onde se encontra a percepção corporal ${ }^{6}$.

A hemiplegia pode causar dependência de dispositivos auxiliares para deambulação e em outras atividades de vida diária, fazendo com que ocorra um conjunto de características que levem à chamada "Síndrome de Pusher" (SP), caracterizada pelo ato de empurrar o corpo fortemente para o lado hemiplégico, causando a perda de consciência corporal, distorcendo a imagem corporal do paciente. As consequências vão desde alterações no equi- líbrio estático em diferentes posiçóes, desequilíbrio da marcha a negligência da metade corporal comprometida. $\mathrm{O}$ ato de empurrar o lado afetado ocorre nas posiçóes sentada, em pé e realizando transferências, sendo que o paciente impóe resistência na tentativa passiva de correção postural ${ }^{2}$.

Da mesma forma, a sensibilidade, a visão, a audição, a expressão facial e a fala podem estar ausentes, ou acentuadamente diminuídas, pois o paciente não percebe os estímulos que chegam de seu lado afetado, demonstrando abandono do lado hemiplégico ${ }^{7-9}$.

Esta perda da imagem corporal pode afetar o paciente de modo que ele se torne cada vez mais dependente de seu cuidador e de sua família, pois quando seu corpo é sustentado para o lado não-hemiplégico, ocorrem deformidades corporais (escolioses, encurtamentos de musculatura dos membros superiores e inferiores etc.), as quais serão difíceis de retroceder, sem antes retomar sua imagem corporal ${ }^{10-12}$.

O paciente acredita estar alinhado, por isso resiste à correção passiva do terapeuta, comprovando que seu esquema corporal encontra-se completamente alterado. Neste sentido, os recursos terapêuticos utilizados para o tratamento do alinhamento corporal incluem o treinamento motor, como a mudança de posiçóes de sentado para em pé; colocação de uma cadeira diante de seus olhos, exatamente no meio do seu campo de visão, para criar uma percepção de linha média; treinamento de marcha com terapeuta atrás do paciente, com as mãos facilitando o movimento no quadril, para não estimular um padrão anormal; estímulos visuais com espelhos, para conscientizar a postura correta; treinamento de equilíbrio e fortalecimento muscular com o uso de bola suíça; e estimulação elétrica neuromuscular para aumentar a amplitude do movimento de extensão da metade corporal afetada pela lesão encefálica ${ }^{13-17}$.

O objetivo da pesquisa foi avaliar o grau de comprometimento da percepçáo corporal em pacientes pós-AVC, através da Scale for Controversive Pushing (CSCP).

\section{MÉTODO}

\section{Amostra}

A pesquisa foi realizada nas dependências da Clínica-Escola de Fisioterapia (CEF) do Centro Uni- 
versitário Univates/Lajeado-RS nos meses de agosto a outubro de 2012, após ter sido aprovada por Comitê de Ética em Pesquisa, mediante protocolo CAAE: 03664112.2.0000.5310. Fizeram parte da população, todos os pacientes em atendimento na CEF da Univates no semestre 2012B com diagnóstico clínico de AVC, de qualquer faixa etária e sexo, que aceitassem participar, mediante assinatura do Termo de Consentimento Livre e Esclarecido, totalizando 18 pacientes. Foram excluídos apenas os pacientes que náo permaneciam em ortostase de forma independente, num total de cinco sujeitos, ficando a amostra final constituída por 13 pacientes. Objetivando um melhor esclarecimento antes do início das coletas, os pacientes e/ou responsáveis foram informados quanto aos propósitos e procedimentos técnicos a serem adotados.

\section{Procedimento}

Como protocolo de avaliação, foi aplicado a CSCP. Esta escala consiste da avaliação do paciente em três etapas: A) a primeira é apenas a observação do paciente pelo pesquisador, para verificar a postura corporal nas posiçóes sentada e em pé (se simétrica ou assimétrica); B) na segunda, é observado se o paciente permanece com abdução e/ou extensão das extremidades corporais não-paréticas ao movimentar-se, com o objetivo de buscar equilíbrio, sentado e em pé; C) e na terceira é realizada uma resistência passiva pelo pesquisador lateralmente ao tórax do paciente (que é levemente empurrado para o lado), para correção da inclinação corporal (caso houver) em pé e sentado. Em cada uma destas etapas (A, B e C), dependendo das reaçóes do paciente, o resultado é anotado recebendo uma pontuação, que varia de zero a dois, sendo zero uma percepção corporal conservada e dois a perda da mesma. A pontuação final do teste pode variar entre zero e seis, sendo o resultado mais próximo de zero considerado percepção corporal normal, mais próximo de três, alteração dessa percepção e mais próximo de seis, a perda da mesma.

\section{Análise Estatística}

Foi realizada uma análise de regressão múltipla considerando a pontuação como variável dependente e o tempo de ocorrência do AVC e a idade, as variáveis independentes.
A média de idade dos homens e mulheres foi comparada pelo teste $t$, o tempo de ocorrência do AVC e a pontuação foram comparadas entre os dois gêneros pelo teste não paramétrico de Mann-Whitney. Foi considerado significativo $\mathrm{P}<0,05$.

\section{RESULTADOS}

\section{Dados demográficos dos pacientes}

Oito pacientes do sexo masculino e cinco do sexo feminino, cujas idades variaram entre 36 e 73 anos (média de 54,5 anos), sendo $46 \%$ idosos. O tempo de doença variou de seis meses a 33 anos (média de 16,7 anos), três pacientes apresentaram AVC do tipo hemorrágico, oito do tipo isquêmico $(62 \%)$ e dois não sabiam informar e não dispunham de exames para verificação. A metade corporal direita estava comprometida em seis pacientes (47\%) e a esquerda, em sete pacientes, representando $53 \%$ da amostra (Tabela 1).

Não foi encontrada influência significativa das variáveis independentes, idade $(\mathrm{p}=0,0730)$ e tempo de ocorrência do AVC ( $\mathrm{p}=0,6164)$ na pontuação (variável dependente), ( $\mathrm{F}=2,0334, \mathrm{p}=0,182)$.

Os homens apresentam significativamente $(\mathrm{p}=$ 0,0052) em média $(62,3)$ mais idade que as mulheres $(44,8)$. Com relação ao tempo de ocorrência do AVC, ocorre também diferença significativa $(\mathrm{p}=0,0404)$, os homens apresentam em média $(84,1)$ maior tempo de ocorrência do AVC que as mulheres $(\mathrm{p}=16,8)$. A pontuação não apresentou diferença significativa entre homens e mulheres $(\mathrm{p}=0,1243)$.

Cinco dos treze pacientes participantes do estudo (P9, P10, P11, P12, P13) apresentaram déficit de alinhamento corporal, dois com inclinação para a direita e três para a esquerda (de acordo com a metade corporal comprometida pela lesão encefálica). Um dos pacientes (P13), do sexo masculino, de 68 anos de idade com AVC hemorrágico e metade corporal esquerda afetada, com dois anos e nove meses de doença, foi o que apresentou a maior pontuação na escala, obtendo nota cinco, com significativa perda da simetria corporal, estando apenas um ponto abaixo da perda total desta simetria. $O$ único participante (P1) que apresentou pontuação zerada (percepção corporal íntegra), também do sexo masculino, com 49 anos de idade, está com 10 meses de doença e tem a 
Tabela 1

Dados demográficos e resultados encontrados

\begin{tabular}{ccccccc}
\hline Paciente & Sexo & $\begin{array}{c}\text { Idade } \\
\text { (anos) }\end{array}$ & $\begin{array}{c}\text { Tipo de } \\
\text { AVC }\end{array}$ & Hemiplegia & $\begin{array}{c}\text { Tempo } \\
\text { doença } \\
\text { (meses) }\end{array}$ & $\begin{array}{c}\text { Pontos } \\
\text { CSCP }\end{array}$ \\
\hline P1 & M & 49 & Náo sabe & D & 10 & 0 \\
P2 & M & 68 & I & E & 68 & 0,25 \\
P3 & F & 42 & I & D & 16 & 0,25 \\
P4 & F & 38 & H & E & 32 & 0,5 \\
P5 & F & 57 & I & E & 24 & 1,25 \\
P6 & F & 36 & I & D & 6 & 1,5 \\
P7 & F & 51 & I & D & 6 & 1,5 \\
P8 & M & 66 & Não sabe & E & 396 & 1,75 \\
P9 & M & 49 & H & E & 33 & 2,25 \\
P10 & M & 73 & I & D & 15 & 2,5 \\
P11 & M & 62 & I & D & 49 & 2,5 \\
P12 & M & 64 & I & E & 69 & 3 \\
P13 & M & 68 & H & E & 33 & 5 \\
\hline
\end{tabular}

Legenda: M (masculino); F (feminino); AVC (acidente vascular encefálico); I (isquêmico); H (hemorrágico); CSPC (Scale for Controversive Pushing)

metade corporal direita afetada. Esses resultados ressaltam que provavelmente o tempo de doença, a idade, o sexo e a metade corporal comprometida pela lesão encefálica, não se relacionam com a alteração da percepção corporal. Os demais sujeitos não apresentaram alteração da percepção corporal, segundo critérios da CSCP. Dos participantes com AVC isquêmico, cinco apresentaram a metade corporal esquerda comprometida $(62,5 \%)$ e três, a direita (37,5\%). Já no AVC hemorrágico, todos os participantes apresentaram a metade corporal esquerda comprometida pela lesão encefálica, conforme descrito na Tabela 1 .

\section{DISCUSSÃO}

O presente estudo aborda as alteraçóes na percepção corporal em pacientes pós- AVC, distúrbio conhecido cientificamente como síndrome de Pusher (SP). Revisão detalhada da literatura mostra estudos que enfocam este espectro de doença $a^{2,8-11,13-16}$.

Considerando a elevada incidência de novos casos de AVC a cada ano, com importante impacto na qualidade de vida destas pessoas, bem como, controvérsias entre a frequência relativa da SP em publicaçooes científicas, sugere-se que estudos adicionais, com aprimoramento metodológico sejam realizados.
Chama-se a atenção para a importância de se tornarem públicas as principais características da SP, para que os profissionais saibam lidar com este tipo de paciente $^{12}$, pois ainda há divergência entre autores sobre as consequências funcionais para pacientes que apresentam alteração da percepção corporal, decorrente da SP pós$-\mathrm{AVC}^{8-16}$.

Autores relatam que após estudo realizado com 14 hemiparéticos dependentes ou não, avaliados quanto ao alcance funcional e simetria na descarga de peso, não foi verificada diferença significativa no alcance funcional entre os pacientes simétricos e assimétricos. Porém, quando a descarga de peso é maior para o lado não afetado (contrário a SP), o alcance funcional é progressivamente maior, quanto menor for essa assimetria ${ }^{17}$.

Contrariamente aos autores descritos anteriormente, pesquisa descreve que a assimetria corporal, ou seja, a perda da percepção corporal está infinitamente relacionada à perda da função do paciente, afetando a relação entre cada parte do corpo e o meio ambiente, podendo causar também a dependência de dispositivos auxiliares para deambulação e/ou prevenir deformidades ósseas, o que limita as atividades de vida diárias do paciente, diminuindo suas funçốes ${ }^{11}$. Simetria e transferência de peso 
correlacionam-se ao bom desempenho de funcionalidade na vida dos pacientes, sendo que quanto melhor a simetria e a transferência de peso, mais independente é o paciente e melhor é a sua marcha, quando ela existe. A boa simetria corporal favorece também no equilíbrio ${ }^{11,18}$.

Com relação à idade da ocorrência de AVC na população, estudo realizado em Campina Grande/PB, com uma amostra de 350 pacientes com AVC, aponta que $51 \%$ dos sujeitos acometidos eram do sexo masculino, a grande maioria de idosos, com AVC decorrente de processo isquêmico em $57 \%{ }^{19}$.

Outro estudo realizado em Passo Fundo/RS revelou que há prevalência de AVC em homens (72,09\%) e em idosos $(44,10 \%)$, sendo o tipo mais comum também o isquêmico $(86,10 \%)$, corroborando nossos achados ${ }^{20}$.

Alguns autores acreditam que a disfunção da percepção corporal esteja relacionada com as compensaçóes necessárias pelo paciente para justamente alcançar funcionalidade com seu membro afetado ${ }^{21-23}$.

Utilizando a CSCP para confirmar o diagnóstico da síndrome, profissionais planejaram tratamento fisioterapêutico aquático como forma de melhorar a desordem muscular proveniente da assimetria corporal. Concluíram que a hidroterapia, baseada nos métodos de Hallwick e Bad Ragaz, foi eficaz para o realinhamento e busca da simetria de tronco, sendo adotados exercícios de reforço e alongamento muscular no processo de reabilitação ${ }^{13}$.

$\mathrm{Na}$ SP os pacientes realizam esforço a fim de buscar o centro de equilíbrio, o qual permanece alterado neste tipo de paciente, porém, outros autores afirmam que esse centro não é alcançado, permanecendo o paciente na posição inclinada ${ }^{14}$. Pode ser encontrada, tanto em pacientes com hemisfério cerebral esquerdo afetado, como no direito ${ }^{10}$, entretanto, para a primeira autora a citar a síndrome, apenas pessoas com AVC no hemisfério direito seriam acometidas pelos sintomas ${ }^{24}$.

Apesar do aumento do número de estudos relacionados a esta síndrome, ainda existe muita discordância entre os pesquisadores quanto à prevalência, fisiopatologia e tratamento desta alteração ${ }^{15}$. Neste sentido, estudos acerca da SP devem ser amplamente divulgados e incentivados, visto que esta condição incapacitante é ainda pouco conhecida entre profissionais da área da saúde ${ }^{25}$. Ademais, estudos adicionais são necessários para esclare- cer diversas lacunas no conhecimento sobre a SP, tanto de sua relação com déficits neurológicos e incapacidade funcional, quanto de seu mecanismo causador e métodos de reabilitação.

\section{CONCLUSÃO}

Considerando o objetivo da pesquisa, de identificar o grau de comprometimento da percepção corporal em pacientes pós-AVC, os resultados apontam que a SP é um distúrbio presente nestes sujeitos e que a Scale for Controversive Pushing (CSPS) é um protocolo de fácil aplicação e que favorece a identificação e gravidade desta alteração.

\section{REFERÊNCIAS}

1.Cappelari MM, Grave MTQ. Avaliação do comprometimento sensório-motor de pacientes com diagnóstico de acidente vascular encefálico (AVE) atendidos na clínica-escola de Fisioterapia da Univates. Rev. Destaques Acadêmicos 2012;4:61-72.

2.O'Sullivan SB. Acidente Vascular Encefálico. In: O'Sullivan SB, Schmitz TJ. Fisioterapia: Avaliaçăo e Tratamento. 2nd ed. Barueri: Manole; 2004, p.51964.

3.Correia ACS, Silva JDS, Silva LVC, Oliveira DA, Cabral ED. Crioterapia e cinesioterapia no membro superior espástico no acidente vascular cerebral. Fisio Mov 2010; 23:555-63.

4.Brito ES, Rabinovich EP. Desarrumou tudo! O impacto do acidente vascular encefálico na família. Sau e Soc 2008;17:153-69.

http://dx.doi.org/10.1590/S0104-12902008000200015

5.Felice TD, Santana LR. Recursos fisioterapêuticos (crioterapia e termoterapia) na espasticidade: revisão de literatura. Rev Neurocienc 2009;17:57-62.

6.Davies PM. Steps to follow: a guide to the treatment of adult hemiplegia. New York: Springer 1985.

http://dx.doi.org/10.1007/978-3-642-96833-4

7.Silveira LCL. Os Sentidos e a Percepção. In: Lent R. Neurociência da mente e do Comportamento. Rio de Janeiro: Guanabara Koogan; 2008. p. 134-81.

8.Karnath HO, Broetz D. Understanding and Treating "Pusher Syndrome". Physical Therapy 2003;12:1119-25.

9.Baccini M, Paci M, Nannetti L, Biricolti C, Rinaldi LA. Scale for Contraversive Pushing: Cutoff Scores for Diagnosing "Pusher Behavior" and Construct Validity. Physical Therapy 2008;88:947-55.

http://dx.doi.org/10.2522/ptj.20070179

10.Johannsen L, Broetz D, Naegele T, Karnath HO. "Pusher syndrome" following cortical lesions that spare the thalamus. J Neurol 2006;253:455-63. http://dx.doi.org/10.1007/s00415-005-0025-7

11.Voo MC, Oliveira TP, Piemonte MEP. Diretrizes para avaliaçâo e tratamento fisioterapêutico da Síndrome de Pusher: estudo de caso. Fisioter Pesq 2011;18:323-28.

12.Góis CMS, Araújo MCNV, Silva KC, Araújo ATC. Avaliação do Conhecimento dos Fisioterapeutas Neurofuncionais acerca da Síndrome de Pusher. Rev 
Neurocienc 2011;19:595-601.

13.Meneghetti CHZ, Basqueira C, Fioramonte C, Ferracini JLC. Influência da fisioterapia aquática no controle de tronco na síndrome de Pusher: estudo de caso. Fisioter. Pesqui. [online] 2009;16:269-73.

14.Santos-Pontelli TEG, Pontes-Neto OM, Colafêmina JF, Araújo DB, Santos AC, Leite JP. Controle postural na síndrome de Pusher: influência dos canais semicirculares laterais. Rev Bras Otorrinolaringol 2005;71:448-52. http://dx.doi.org/10.1590/S0034-72992005000400008

15.Palmini S, Costa J, Grave M. Síndrome de Pusher em pacientes com AVC e sua associação com gravidade clínica e dependência funcional. Rev Neurocienc 2013;21:69-76.

http://dx.doi.org/10.4181/RNC.2013.21.789.8p

16.Santos-Ponteli TEG, Leite JP, Pontes-Neto OM. Avaliação e análise da frequência da Síndrome de Pusher (Síndrome do Empurrador) em pacientes com AVC. Rev Neurocienc 2013;21:12-3.

http://dx.doi.org/10.4181/RNC.2013.21.789ed.2p

17.Pereira LC, Botelho AC, Martins EF. Correlação entre simetria corporal na descarga de peso e alcance funcional em hemiparéticos crônicos. Rev Bras Fisiot 2012;10:218-225.

18. Trindade APNT, Barboza MA, Oliveira FB, Borges APO. Influência da simetria e transferência de peso nos aspectos motores após Acidente Vascular Cerebral. Rev Neurocienc 2011;19:61-7.

19.Lavor IG, Agra G, Nepomuceno CM. Perfil dos casos de Acidente Vascu- lar Cerebral registrados em uma instituição pública em Campina Grande-PB. Campina Grande 2011;12:s/p.

20.Mazzola D, Polese JC, Schuster RC, Oliveira SG. Perfil dos pacientes acometidos por Acidente Vascular Encefálico assistidos na Clínica de Fisioterapia Neurológica da Universidade de Passo Fundo. RBPS 2007;20:22-7.

http://dx.doi.org/10.5020/18061230.2007.p22

21.Van Peppen RPS, Kortsmit M, Lindeman E, Kwakkel G. Effects of visual feedback therapy on postural control in bilateral standing after stroke: a systematic review. J Rehabil Med 2006;38:3-9.

http://dx.doi.org/10.1080/16501970500344902

22. Teixeira-Salmela LF, Lima RCM, Lima LAO, Morais SG, Goulart F. Assimetria e desempenho funcional em hemiplégicos crônicos antes e após programa de treinamento em academia. Rev Bras Fisioter 2005;9:227-33.

23. Genthon N, Rougier P, Gissot AS, Froger J, Pélissier J, Pérennou D. Contribution of each lower limb to upright standing in stroke patients. Stroke 2008;39:1793-9.

http://dx.doi.org/10.1161/STROKEAHA.107.497701

24.Davies PM. Steps to folow: a guide to the treatment of adult hemiplegia. New York: Springer; 1985,300p.

25.de Haart M, Geurts AC, Huidekoper SC, Fasotti L, van Limbeek J. Recovery of standing balance in postacute stroke patients: a rehabilitation cohort study. Arch Phys Med Rehabil 2004;85:886-95.

http://dx.doi.org/10.1016/j.apmr.2003.05.012 